\title{
Mapping UHF RFID Tags with a Mobile Robot using a 3D Sensor Model
}

\author{
Ran Liu, Artur Koch, and Andreas Zell
}

\begin{abstract}
Recently, researchers showed growing interest in utilizing UHF Radio-Frequency Identification (RFID) technology for localizing tagged items with mobile robots in industrial scenarios. In this paper we present a novel three-dimensional (3D) probability sensor model of RFID antennas in the context of mapping passive RFID tags with mobile robots. The proposed 3D sensor model characterizes both detection rates and received signal strength (RSS). Compared to 2D-sensor model based approaches, the 3D model gains a higher mapping accuracy for 2D position estimation. Specially, with this sensor model, we are able to localize the tags in 3D by integrating the measurements from a pair of RFID antennas mounted at different heights of the robot. Furthermore, by integrating negative information (i.e., non-detections), the 3D mapping accuracy can be improved. Additionally, we utilize KLD-sampling to reduce the number of particles for our specific application, so that our algorithm can be performed online. Indoor experiments with a Scitos G5 robot demonstrate the effectiveness of our approach. We also provide the datasets of this work for download.
\end{abstract}

\section{INTRODUCTION}

In recent years, robot-assisted applications in commercial surroundings such as supermarkets, warehouses, and logistic centers have attracted more and more attention. For these environments, there is an increasing use of UHF RFID tags for labeling products, assets, or equipment. If a robot is equipped with an RFID reader in such environments, automated inventory becomes possible. Compared to the traditional inventory process, the products can not only be itemized but also be localized by a sequence of RFID readings [1], [2], [3] provided by the mobile agent.

However, mapping of RFID tags is quite challenging: The propagation of radio waves is hard to model due to many influencing environmental effects (e.g., reflection, diffraction, or absorption). Nevertheless, measurements (e.g., readability or RSS values) reported by the reader highly depend on the relative position of the tag to the antenna.

Therefore, we propose a 3D RFID sensor model for mapping RFID tags. Unlike the existing 2D sensor models, our 3D sensor model characterizes the detection behavior of the RFID antenna with respect to the 3D positions of RFID tags. As visualized in Fig. 1, we can observe that measurements (the detection counts and mean RSS values) from RFID readers, not only depend on the relative $x y$ displacement but also rely on the height of the tag w.r.t. the antenna coordinate frame. The contribution of this paper is threefold: First, by applying our 3D sensor model, 2D position estimation

R. Liu and A. Koch are with the Chair of Cognitive Systems, headed by Prof. A. Zell, Computer Science Department, University of Tübingen, Sand 1, D-72076 Tübingen, Germany \{ran.liu, artur.koch, andreas.zell@uni-tuebingen.de

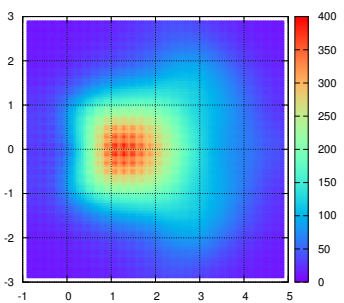

(a) Detection counts at $0 \mathrm{~m}$

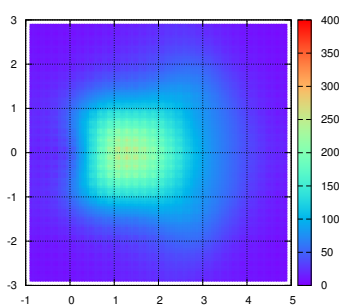

(c) Detection counts at $0.8 \mathrm{~m}$

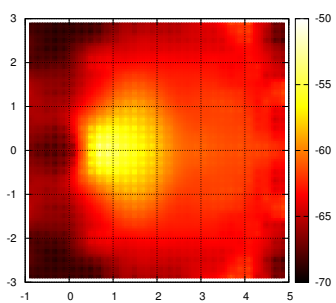

(b) Mean RSS at $0 m$

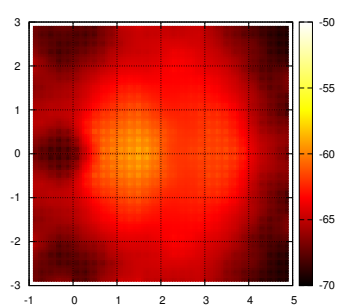

(d) Mean RSS at $0.8 \mathrm{~m}$
Fig. 1. Visualization of the detection counts (left) and mean RSS values (right) of an RFID antenna w.r.t. the position of RFID tags at full reader transmission power.

can be improved, as compared to our previous $2 \mathrm{D}$ sensor model. Since the position of the antennas is fixed in our application, by using only one antenna, we are not able to determine the height of RFID tags unambiguously due to the symmetrical radiation property of antennas. Therefore, as a second contribution, we show how the ambiguity can be resolved and the mapping error can be reduced by a pair of RFID antennas installed at different heights of the robot. Third, we show that the 3D position estimation can be further improved by incorporating non-detections for our specific stereo antenna configuration. Moreover, we employ KLDsampling to reduce the number of particles. This enables our algorithm to perform the mapping task in real-time.

The rest of this paper is structured as follows: After an overview of the related work in Sect. II, we introduce the Bayesian approach to localize RFID tags with sensor models in Sect. III. Then, we describe the details of our sensor model in Sect. IV, and Sect. V explains how to use KLDsampling to adapt the particle set. In Sect. VI, we describe how to apply non-detections to improve mapping accuracy. Finally, we present our experimental results in Sect. VII, and conclude this paper in Sect. VIII.

\section{RELATED WORK}

A variety of approaches concerning the mapping of RFID tags in the context of mobile robots are proposed in literature. Hähnel et al. [1] were the first to utilize a sensor model to determine the location of RFID tags with a mobile robot, 
whose position is estimated by the laser-based FastSLAM. Vorst et al. [4] showed that sensor models can be learned in a semi-autonomous way during normal navigation of the robot. Joho et al. [2] incorporated RSS values as well as tag detection rates into their sensor models to improve mapping accuracy of RFID tags.

Several researchers have proposed to fuse other sensors, like laser range finders, or visual sensors with RFID measurements, to improve the mapping accuracy. Germa et al. [5] combined RFID measurements with visual information to track people with a mobile robot in a crowded environment. Rohweder et al. [6] utilized the spatial structure of the environment to further reduce the estimation error of passive RFID tags. Deyle et al. [7] generated a 2D image which characterizes the spatial distribution of RSS for an RFIDtagged object by rotating mobile antennas. By aligning this RSS image with a color image and 3D laser data, they are able to localize objects in 3D space.

\section{MAPPING RFID TAGS}

In this paper, the positions of the transponders are estimated by a Bayesian inference similar to [1], [2], [4]. Formally, we denote the number of RFID tags around the environment by $L . \mathrm{m}_{1: t}=\left\{\mathrm{m}_{1}, \cdots, \mathrm{m}_{t}\right\}$ represents the stream of measurements gathered by the mobile robot at positions $\mathrm{x}_{1: t}=\left\{\mathrm{x}_{1}, \cdots, \mathrm{x}_{t}\right\}$ until time $t$. Here $\mathrm{m}_{t}$ consists of the measurement queried by the RFID antennas at time $t$ : $\mathrm{m}_{t}=\left\{\mathrm{m}_{t}^{(1)}, \cdots, \mathrm{m}_{t}^{(A)}\right\}$, where $A$ is the number of antennas attached to the RFID reader. The measurement $\mathrm{m}_{t}^{(a)}$ is expressed as a sequence $\mathrm{m}_{t}^{(a)}=\left\{\mathrm{m}_{t, 1}^{(a)}, \cdots, \mathrm{m}_{t, L}^{(a)}\right\}$, where $\mathrm{m}_{t, j}^{(a)}$ represents the measurement of tag $j$ observed from antenna $a$ at time step $t$. In addition, let $\delta_{t}^{(a)}$ denote the position of the tag w.r.t. antenna $a$ 's coordinate system at time $t: \delta_{t}^{(a)}=(x, y, z)$. We can compute $\delta_{t}^{(a)}$ by simply transforming the robot coordinates with a fixed transformation matrix $\mathbf{C}_{a}^{r}$, since the position of the antennas is fixed on the robot, as shown in Fig. 2(a) and 2(b).

To estimate the location $\mathrm{l}_{j}$ of $\operatorname{tag} j$, we need to know the posterior probability $p\left(\mathrm{l}_{j} \mid \mathrm{m}_{1: t}, \mathrm{x}_{1: t}\right)$. In our case, we consider the position of the tag in three dimensions, thus $\mathrm{l}_{j}=\left(x_{j}, y_{j}, z_{j}\right)$. According to the Bayesian theory and the Markov assumption, $p\left(\mathrm{l}_{j} \mid \mathrm{m}_{1: t}, \mathrm{x}_{1: t}\right)$ can be rewritten as:

$$
\begin{aligned}
p\left(\mathrm{l}_{j} \mid \mathrm{m}_{1: t}, \mathrm{x}_{1: t}\right) & =\eta_{1 \ldots t} \prod_{i=1}^{t} p\left(\mathrm{~m}_{i} \mid \mathrm{l}_{j}, \mathrm{x}_{i}\right) p\left(\mathrm{l}_{j}\right) \\
& =\eta_{1 \ldots t} \prod_{i=1}^{t} \prod_{a=1}^{A} p\left(\mathrm{~m}_{i, j}^{(a)} \mid \mathrm{l}_{j}, \mathrm{x}_{i}\right) p\left(\mathrm{l}_{j}\right) \\
& =\eta_{1 \ldots t} \prod_{i=1}^{t} \prod_{a=1}^{A} p\left(\mathrm{~m}_{i, j}^{(a)} \mid \delta_{t}^{(a)}\right) p\left(\mathrm{l}_{j}\right)
\end{aligned}
$$

The formulas above allow us to iteratively estimate the position of a tag by applying the sensor model based on observed measurements. The most important part in Eq. (3) is $p\left(\mathrm{~m}_{i, j}^{(a)} \mid \delta_{t}^{(a)}\right)$, which is called sensor model. It characterizes the likelihood of measurements $\mathrm{m}_{i}$ of a specific tag $j$ at a relative position to the antenna $\delta_{t}^{(a)}$. The formulas above are

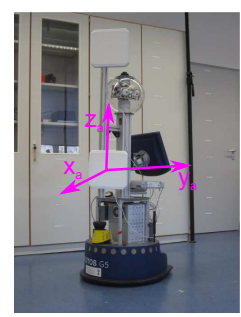

(a) Robot

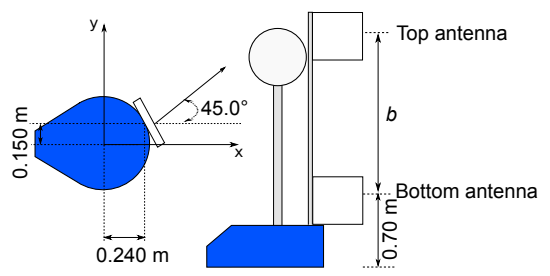

(b) Antenna setup
Fig. 2. (a) The mobile robot used for our experiments and the annotation of the bottom antenna's coordinate system (marked with pink). $x y$-plane is parallel to the ground plane, $z$-axis is perpendicular to $x y$-plane. (b) Antenna setup of our Scitos G5 robot. Vertical (left) and side view (right).

derived under the following assumptions (cf. [1], [3]): the current observation $\mathrm{m}_{t}$ is independent of previous observations $\mathrm{m}_{1: t-1}$; the observation of one tag is independent of other tags in the environment; only the relative displacement between tag and antenna is relevant to our sensor model.

Certainly, these assumptions are too strict in practice. The readability and RSS of a tag are influenced by the neighboring tags [8]. Furthermore, location-specific factors, such as the materials the tags are attached to, obstacles in the environment, orientation between tag and antenna or other radio devices in the environment, have high influence on the behavior of the RFID reader. Thus, many authors applied location-fingerprinting techniques to improve the localization accuracy of mobile agents [9], [10]. These approaches estimate the position of a mobile agent from a set of precollected observations. They showed a slight improvement to model-based approaches [9]. However, the concern of this paper is mapping of RFID tags, which requires an explicit sensor model and it is almost impossible to take all environmental factors into account.

The position of each RFID tag is estimated by an individual particle filter. Each particle filter consists of $N$ samples of position hypotheses: $\mathrm{x}^{(i)}=\left(x^{(i)}, y^{(i)}, z^{(i)}\right)_{1 \leq i \leq N}$ w.r.t. the world coordinate frame and associated weights $w^{(i)}$. The position of the tag is computed by a weighted mean among all particles. The particles are initialized uniformly around the area of the antenna position, at which the tag is first detected. Specifically, $x$ and $y$ coordinates are restricted by the maximum range of the RFID reader and $z$ coordinates are limited by the maximum height of the tags (e.g., $3 \mathrm{~m}$ ).

Theoretically, Eq. 3 allows us to estimate the 3D positions of RFID tags by integrating several detections at different 3D positions. It is worth pointing out that we are not able to unambiguously determine the height of RFID tag if our mobile agent only moves in the $x y$ plane and carries only one antenna or several antennas mounted at the same height. As shown in Fig. 3, the posterior probability density representation over the height of RFID tag obeys a bimodal distribution due to the symmetrical characteristics of the antenna. However, by integrating the measurements from a second antenna, this ambiguity can be resolved, as shown in Fig. 3(c). Particularly, we employ a pair of RFID antennas, which we call stereo antenna configuration in this paper, since the antenna poses only differ in the $z$-coordinate spanning a distance $b$ (called baseline), as shown in Fig. 2 . 


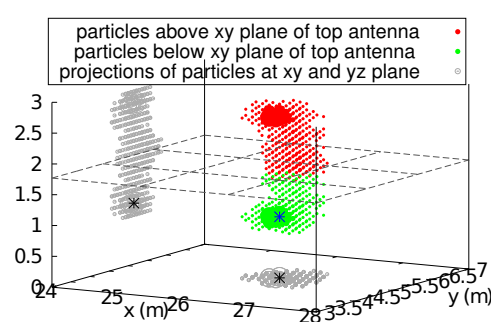

(a) After 20th update with only top antenna.

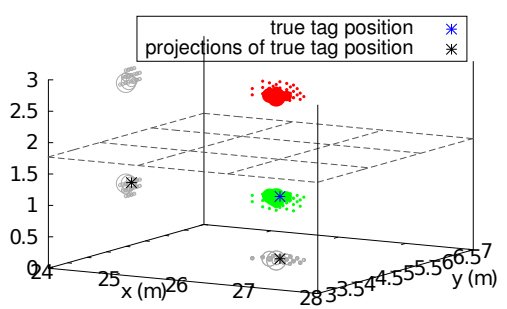

(b) After 30th update with only top antenna.

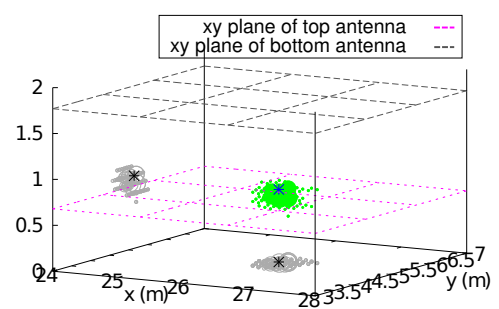

(c) After 30th update with our stereo setup.

Fig. 3. Visualization of 3D Mapping using only top antenna or stereo antenna system. The size of the circles represents the weight of the particles. Particles with too small weights are neglected in our visualization. (a) After processing 20 detections with only top antenna, there is high uncertainty about the position of the tag: the estimation of $z$ is quite ambiguous, but $x y$ estimation is quite well, as can be seen from the projections of the particles. (b) After 30th update, it is more clear to see that the particle filter converges to two regions that are symmetrical to the $x y$ plane of the top antenna. (c) By integrating measurements from a pair of antennas, after 30 measurements, the particles converge to one region and the ambiguity is resolved.

In our implementation, the positions and weights of particles are expressed as double values and kept in memory without compression. In the case of $2 \mathrm{D}$ sensor models, we use $N=1000$ particles, which takes approx. $1 \mathrm{~ms}$ for each measurement update (cf. Sect. VII-D), to achieve a good mapping accuracy. Therefore the algorithm can be run in real time. Approx. 45.7 $\mathrm{MB}$ of memory are required to localize 2000 RFID tags in a store on-line. However, 3D position estimation requires a higher number of particles, due to the additional dimension. We utilize a particle filter with 10000 samples to get a good position estimation. Hence, time complexity and memory consumption increase due to the increased number of particles. To reduce the running time of our mapping module, we utilize KLD-sampling to adapt the number of particles, as detailed in Sect. V.

Since we assume the tags to be static (i.e., tags do not change their positions while the robot traverses the environment), we neither utilize motion information to predict the movement of the particles nor execute resampling for our particles. This strategy is also deployed by Hähnel et al. [1], and Rohweder et al. [6]. For a comparison and discussion of particle filter related resampling/pertubation approaches, we refer to Joho et al. [2] and the dissertation of Vorst [3].

\section{SENSOR MODEL}

The sensor model $p(\mathrm{~m} \mid(x, y, z))$ describes the likelihood of observing a tag at a relative position to the antenna. In our situation, each measurement $\mathrm{m}=(d, s)$ reports two pieces of information: a binary value $d$ which indicates that the tag is detected, and the associated signal strength $s$. The sensor model can be extended as [2]:

$$
p(\mathrm{~m} \mid(x, y, z))=\underbrace{p(s \mid d,(x, y, z))}_{\text {RSS likelihood }} \underbrace{p(d \mid(x, y, z))}_{\text {detection probability }}
$$

Thus, the likelihood of an observation can be modeled as the fusion of two independent probabilities: the probability of receiving the signal strength $s$ at position $(x, y, z)$, and the likelihood of detecting the tag at this position. The latter one alone is called tag detection probability, and the respective model is called detection rate model.

We utilize a sensor model that incorporates RSS values into detection rates, which was introduced by Joho et al. [2] to improve the mapping accuracy of RFID tags. In this paper, we refer to this sensor model as combined model. Sensor models are usually represented as discretized grid cells. Joho et al. [2] assumed that the RSS values inside grid cells obey a gaussian distribution with a mean $\mu_{(x, y, z)}$ and a standard deviation $\sigma_{(x, y, z)}$. Based on this assumption, the likelihood of observing the given RSS in that grid cell can be calculated by:

$$
p(s \mid d,(x, y, z))=\frac{1}{\sqrt{2 \pi} \sigma_{(x, y, z)}} \exp \left(-\frac{\left(s-\mu_{(x, y, z)}\right)^{2}}{2 \sigma_{(x, y, z)}^{2}}\right)
$$

We use the semi-autonomous approach proposed by Vorst et al. [4] to learn our sensor model in this paper. The RFID measurements are recorded as well as the poses of the robot while the robot is manually controlled to traverse the environment during an exploration phase. After this stage, an off-line training step is performed to learn the sensor model: At each RFID detection pose, the robot transforms the global positions of the reference tags to the antenna's local coordinate frame and counts the non-detections $n_{x, y, z}^{-}$and the detections $n_{x, y, z}^{+}$in each grid cell $(x, y, z)$. The detection probability $p(d \mid(x, y, z))$ is computed by:

$$
p(d \mid(x, y, z))=\frac{n_{x, y, z}^{+}}{n_{x, y, z}^{-}+n_{x, y, z}^{+}}
$$

\section{ADAPTING THE NUMBER OF PARTICLES}

Fox [11] first used KLD-sampling to adapt the particle size for robot localization. KLD-sampling is able to choose the number of particles dynamically w.r.t. the current state of approximation. At each update step, it determines the number of particles that is required for a good approximation, so that we have a probability of $1-\delta$ to make sure the error between the posterior and our estimation is limited by $\varepsilon$. The desired number of particles $n_{\chi}$ is determined by:

$$
n_{\chi}=\frac{1}{2 \varepsilon} \chi_{k-1,1-\delta}^{2}
$$

here $\chi_{k-1,1-\delta}^{2}$ is the $1-\delta$ quantile of the Chi-Square distribution with $k-1$ degrees of freedom. Details about the computation of $\chi_{k-1,1-\delta}^{2}$ can be found in [11], [12].

We use a specialized version of the KLD-algorithm. In our application the tags are assumed to be static, and we therefore neglect resampling and prediction. As a result of this, the importance weights of the particles in the predictive distribution are not uniform after the application of updates. Therefore, after sampling a particle from the predictive 
belief, we keep track of the already chosen samples to not integrate them multiple times into the adapted distribution, which otherwise would lead to a corruption of the posterior. For that reason, the number of sampled particles $n_{\chi}$ differs from the actual number of particles $k_{\chi}$ that are selected for the adapted distribution, since duplicate particles are skipped. To keep diversity in our distribution, we introduce another parameter $k_{\chi \min }$ as a minimum threshold for the desired number of particles. As a result, we get a static Monte-Carlo based representation of our target domain, where particle numbers adapt to the current state of the estimation.

\section{UTILIZING NEGATIVE INFORMATION}

Negative information has been successfully applied for object tracking [13] and localization [14] of mobile robots. In this paper, we treat non-detections as negative information for our stereo antenna configuration to improve 3D mapping accuracy. Detections and non-detections are regarded as mutually exclusive events in this paper, therefore the nondetection model $p\left(m^{-} \mid(x, y, z)\right)$ is computed as:

$$
p\left(m^{-} \mid(x, y, z)\right)=1-p(d \mid(x, y, z))
$$

Non-detections give you an evidence that the tag is absent in a certain area, which may be useful to infer the potential location of the tag. However, it is difficult to balance detections and non-detections in practice. This is because, on the one hand, the particles often converge to a region with low detection likelihood due to false negatives of non-detections, which is shown by Vorst [3]; on the other hand, incorporating non-detections requires more computational time. To compensate for that, we only consider non-detections when the specific tag is detected by at least one antenna in our stereo antenna setup. Particularly, if antenna $a^{+}$detects the tag while $a^{-}$does not, we additionally apply the nondetection sensor model for antenna $a^{-}$. The idea of this is straightforward. Applying negative information may devalue the particles with high likelihood due to false negatives, but by integrating the non-detection model only in case of a detection, we minimize the false-negative count.

\section{EXPERIMENTAL RESULTS}

\section{A. Setup}

In this paper, a Scitos G5 mobile robot from Metralabs, as shown in Fig. 2(a), served as the experimental platform. The robot is installed with a laser range finder (SICK S300) with $240^{\circ}$ field of view, and a UHF RFID reader (Impinj Speedway R1000). The reader is able to provide RSS values $(-35 \mathrm{dbm}$ to $-80 \mathrm{dbm})$ and has a maximum read range of up to 8 meters. The experiments were conducted in an indoor environment with an area of approx. $60 \mathrm{~m}^{2}$. Around $65 \mathrm{UHF}$ RFID tags (Alien Technology Squiggle) were attached to the furniture and the walls of the lab at different heights, ranging from 0.2 to 2.5 meters. The positions of all tags were measured manually beforehand to provide ground truth for our experiments.

We manually steered the robot at different trajectories through the lab with a maximum velocity of $0.2 \mathrm{~m} / \mathrm{s}$. The robot traveled around $1.5 \mathrm{~km}$ with a duration of approx.
TABLE I

2D MAPPING ERRORS (IN CENTIMETERS) UNDER INFLUENCE OF DIFFERENT SENSOR MODELS AND VARIOUS SETUPS OF ANTENNAS.

\begin{tabular}{|c|c||c|c||c|c|}
\hline \multirow{2}{*}{$\begin{array}{c}\text { antenna } \\
\text { config. }\end{array}$} & model & \multicolumn{2}{c|}{ detection rate model } & \multicolumn{2}{c|}{ combined model } \\
\cline { 3 - 6 } & mean \pm std.dev. & median & mean \pm std.dev & median \\
\hline \multirow{2}{*}{ Bottom } & 2D & $39.0 \pm 20.8$ & 38.0 & $35.7 \pm 24.4$ & 29.8 \\
& 3D & $39.1 \pm 21.8$ & 38.0 & $33.1 \pm 20.8$ & 32.7 \\
\hline \multirow{2}{*}{ Top } & 2D & $38.2 \pm 18.6$ & 37.1 & $40.1 \pm 22.5$ & 37.1 \\
& 3D & $36.2 \pm 17.2$ & 37.1 & $35.3 \pm 17.2$ & 34.7 \\
\hline \multirow{2}{*}{ Both } & 2D & $32.6 \pm 13.9$ & 35.5 & $26.2 \pm 16.1$ & 23.7 \\
& 3D & $38.4 \pm 18.6$ & 38.0 & $23.4 \pm 15.6$ & 19.9 \\
\hline
\end{tabular}

three hours, and seven log files were recorded at the same time with a wide baseline stereo antenna configuration $(b=1.1 \mathrm{~m})$. Each $\log$ file consists of at least 2000 RFID measurement and the associated true position of the robot, which is estimated by a laser-based Monte Carlo localization algorithm [15]. Totally, approx. 438762 RFID detections were recorded for the wide baseline configuration. The mean mapping accuracy was averaged by validating one tag using the sensor model learned from the rest of the tags. In addition, we recorded three $\log$ files for a middle baseline configuration $(b=0.7 \mathrm{~m})$, and another three for a small baseline configuration $(b=0.3 \mathrm{~m})$ to compare the impact of various baselines on mapping accuracy. The three different configurations of baseline width were obtained by fixing the bottom antenna and only moving the top antenna, as visualized in Fig. 2(b).

\section{B. Evaluation of $2 D$ mapping accuracy}

The performance and accuracy of 2D mapping using different sensor models was evaluated by a first series of experiments. We used a particle number of 1000 for 2D sensor models. These particles were spread uniformly in the $x y$ plane with a fixed height $z$, e.g., $z=1.0 \mathrm{~m}$. For 3D sensor models, we equally distributed 13000 particles at various heights ranging from 0.0 to 2.4 meters with an interval of $0.2 \mathrm{~m}$. Thus, the experimental results under various sensor models are considered to be comparable, since the $x y$ density of particles for the 3D sensor model is identical to the 2D sensor model. We neither performed KLD-sampling nor incorporated negative information in the experiments. The results are listed in Tab. I, in which we combined different sensor models and antenna configurations. Obviously, using both antennas achieves better results than only one antenna for both 2D and 3D sensor models. We also observed that for all experiments conducted with the combined model, the 3D sensor model clearly improves mapping accuracy. For example, with only the top antenna in use, the mean mapping error is reduced from $0.401 \mathrm{~m}$ to $0.353 \mathrm{~m}$ by applying the 3D sensor model. This gives us an improvement of $12.0 \%$ compared to the 2D sensor model.

As a comparison we evaluated the approach presented by Joho et al. [2]. In case of a single antenna, the mean mapping error (i.e., $0.357 \mathrm{~m}$ for bottom antenna only) is higher than the error $(0.29 \mathrm{~m})$ obtained by Joho. The reason is, that in our work the tags are distributed at different heights, while the tags are located roughly at the same height in Joho's work. However, with a pair of antennas and the 3D sensor model, 


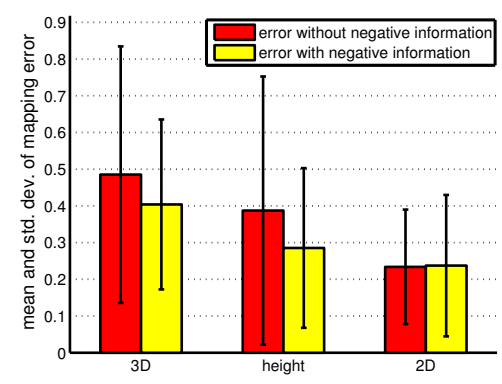

(a)

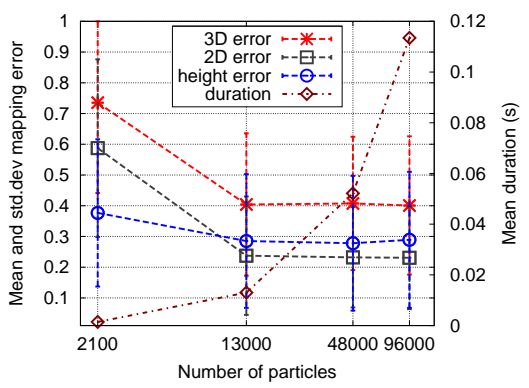

(b)

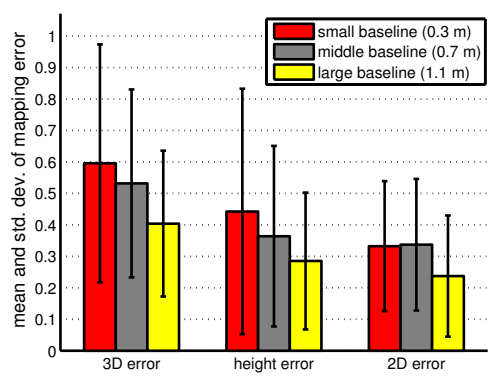

(c)

Fig. 4. Performance evaluation under different parameters. (a) Mapping error with and without negative information; (b) Mean and std. dev. of abs. mapping error versus mean computation time of each detection event under different numbers of particles; (c) Mapping error under various baselines.

we achieve a mean error of $0.234 \mathrm{~m}$ for $2 \mathrm{D}$ localization, which is equivalent or even slightly better than Joho's results.

\section{Influence of negative information}

The influence of negative information on mapping accuracy was investigated in the next experiments. The algorithm was tested using a fixed number of particles $(N=13000)$ without KLD-sampling. As can be seen from Fig. 4(a), which visualizes the mapping results with and without integrating negative information, utilizing negative information improves the mapping accuracy. For example, the mean height error is reduced from $0.38 \mathrm{~m}$ to $0.28 \mathrm{~m}$, which is an improvement of approx. $26.3 \%$. This is due to the fact that we can exploit the stereo setup of our antennas by incorporating negative information: If both antennas detect a tag, we can infer that the tag is probably between the two antennas; otherwise, if only the top antenna detects the tag, it is likely that the tag's location is in the region closer to the top antenna, as described in Sect. VI.

\section{Evaluation of $3 D$ position estimation}

As a third series of experiments, we varied the number of particles $N$ to evaluate the performance of $3 \mathrm{D}$ position estimation, as depicted in Fig. 4(b). We incorporated negative information in these experiments but did not perform KLDsampling. Our algorithm was tested on an Intel Core i52410M @2.3 GHz CPU, with 4 GB RAM. As can be seen from Fig. 4(b), we obtain nearly the same mapping results for $N \geq 13000$, but at the same time the computation time increases drastically. With $N=96000$, we obtain a mean abs. 3D mapping error of $0.40 \mathrm{~m}$. As expected and shown in Fig. 4(b), the mapping errors increase for smaller $N$. Additionally, we noticed that the error in height estimation is usually larger than the error in the $x y$-plane in all experiments. For example, with $N=13000$, the mean height error is approx. $0.28 \mathrm{~m}$, which is much larger than the mean error of $x$ or $y: \frac{0.23}{\sqrt{2}} \approx 0.16 \mathrm{~m}$. This can be explained as follows: $x$ and $y$ positions of the tags are estimated through various measurements collected by the mobile robot that moves in the $x y$ plane, while $z$ is only determined by the difference of two RFID antennas. Thus the deviation of measurements in $x y$ direction is much higher than in the $z$ direction. As a result, we do not get a good $z$-estimation due to the lack of distinct detection samples in the $z$-axis. Another drawback is the required computation time: we need approx. $52 \mathrm{~ms}$ (for $N=48000$ ) to process one tag detection, thus it is impossible to run the algorithm in real time for densely tagged environments, where the robot may receive hundreds of RFID readings per second. However, as shown in Sect. VII-F, by applying KLD-sampling to adapt the number of particles, we are able to run our algorithm online meeting the real-time requirements.

\section{E. Influence of the baseline of the stereo antenna system}

In the next series of experiments, we examined the mapping accuracy under various baselines of our stereo antenna configuration. We did not perform KLD-sampling, but negative information was utilized in these experiments. Due to the physical and technical limitations of our robot, we are not able to set the baseline of the antennas wider than $1.1 \mathrm{~m}$. As can be seen in Fig. 4(c), wider baselines result in better mapping results. With small baselines, the mapping error is notably worse. For example, the height mapping error with the smallest baseline $(0.3 \mathrm{~m})$ is nearly twice as high as compared to the widest baseline $(1.1 \mathrm{~m})$ error. This is because our stereo antenna setup degrades to a single antenna for small baselines, since the difference in the measurements is dominated by the characteristic noise from RF-propagation. In contrast, if the baseline is chosen too wide, there is no overlap between the two RF-fields, and the arrangement degrades to a simple antenna-array with two distinct (single) antennas. Since the possible height on the robot is limited, the second case is of no concern to us under the given configuration (i.e., full transmission power level).

\section{F. KLD-sampling performance evaluation}

To examine the performance of KLD-sampling, a last series of experiments was conducted for varying values of $\varepsilon$ and $k_{\chi \min }$. This technique is also recommended by Fox [11]. We used a bin size of $0.5 m \times 0.5 m \times 0.5 m$ and fixed $\delta$ and $n_{\chi \max }$ to 0.01 and 300000 respectively. Furthermore, we initialized the particle filters with $N=13000$ and integrated negative information for all experiments. For our experiment, the number of particles is reduced below $k_{\chi \min }$ (e.g., 1000) after several updates (e.g, five or ten) of the measurements. Fig. 5 illustrates the 3D mapping accuracy 


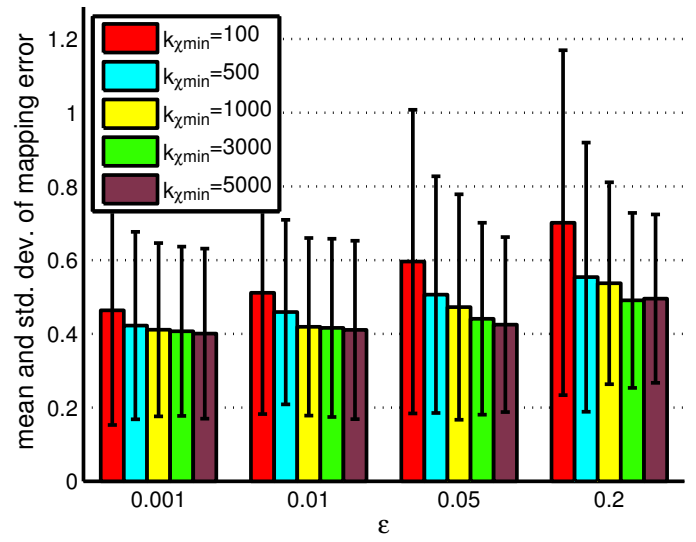

Fig. 5. 3D Mapping error under various combination of $k_{\chi m i n}$ and $\varepsilon$.

under various $\varepsilon$ and $k_{\chi \text { min }}$. Smaller $\varepsilon$ yield better results, while larger $\varepsilon$ lead to worse mapping accuracy. Moreover, it is obvious that larger $k_{\chi \min }$ lead to better results for all $\varepsilon$ values. But in practice, we need to consider the tradeoff between time complexity and accuracy. Mapping with a larger $k_{\chi \min }$ results in a good position approximation. On the contrary, utilizing too many particles requires much more computation time. For example, the difference of mean 3D mapping errors between $k_{\chi \min }=1000$ and $k_{\chi \min }=$ 5000 for $\varepsilon \leqq 0.01$ is less than two centimeters, which is relatively low as compared to the previous mean absolute mapping error of approx. $0.4 \mathrm{~m}$, while the time consumption is doubled at the same time. Hence, it is reasonable for us to choose $k_{\chi \min }=1000$ rather than $k_{\chi \min }=5000$ for $\varepsilon \leqq 0.01$ considering the balance between efficiency and accuracy. In addition, for $k_{\chi \min }=1000$ and $\varepsilon \leqq 0.01$, KLD-sampling only uses approx. $7 \%$ particles of the fixed sampling approach, but yields nearly the same good mapping accuracy.

\section{CONCLUSIONS AND FUTURE WORK}

In this paper, we presented a novel 3D sensor model which considers both detection likelihood and RSS values for localizing UHF RFID tags with a mobile agent. The contribution of this paper is as follows: First, we demonstrated that 2D position estimation can be improved by applying a 3D sensor model. Second, we showed that this sensor model can be used to determine the 3D position of RFID tags by mounting two antennas at different heights. Third, we utilized negative information to further improve mapping accuracy. Fourth, we employed KLD-sampling to be able to perform the mapping task in real-time. The experimental datasets of this paper can be found at our webpage: http://www.cogsys.cs.unituebingen.de/datasets/iros2013.

Comprehensive experiments were conducted to show the effectiveness of our approach. By using our stereo antenna setup and incorporating negative information, we are able to localize RFID tags with a mean abs. localization error of $0.24 m$ in $2 \mathrm{D}$ and $0.40 \mathrm{~m}$ in 3D with $0.28 \mathrm{~m}$ for height. As compared to existing 2D sensor model based approaches, our 3D sensor model improves the 2D mapping accuracy by approx. $12.0 \%$ (from $0.401 \mathrm{~m}$ to $0.353 \mathrm{~m}$ ). Additionally, utilization of negative information reduces the mean abs. mapping error by approx. $26.3 \%$ (from $0.38 \mathrm{~m}$ to $0.28 \mathrm{~m}$ ) for height. Moreover, with KLD-sampling, we are able to save approx. $90 \%$ computation time, while the mean mapping error increases by slightly two or three centimeters.

There are several possible extensions of this work: First, it would be interesting to see if the 3D mapping accuracy can be improved by rotating our stereo antennas or installing more RFID antennas. Another direction could be the integration of an outlier-removal approach, which deals with the ghost detections of RFID tags due to environmental effects on RF propagation, to improve the mapping accuracy.

\section{ACKNOWLEDGEMENT}

We would like to thank Dr. Philipp Vorst for his several years of contribution to the software for our indoor robots.

\section{REFERENCES}

[1] D. Hähnel, W. Burgard, D. Fox, K. Fishkin, and M. Philipose, "Mapping and localization with RFID technology," in Proc. of the 2004 IEEE Int. Conf. on Robotics and Automation (ICRA 2004), USA, 2004, pp. 1015-1020.

[2] D. Joho, C. Plagemann, and W. Burgard, "Modeling RFID signal strength and tag detection for localization and mapping," in Proc. of the 2009 IEEE Int. Conf. on Robotics and Automation (ICRA 2009), Kobe, Japan, May 2009, pp. 3160-3165.

[3] P. Vorst, "Mapping, localization, and trajectory estimation with mobile robots using long-range passive RFID," Ph.D. dissertation, University of Tübingen, Tübingen, Germany, August 2011.

[4] P. Vorst and A. Zell, "Semi-autonomous learning of an RFID sensor model for mobile robot self-localization," in European Robotics Symposium 2008, ser. Springer Tracts in Advanced Robotics, vol. 44/2008. Springer Berlin/Heidelberg, February 2008, pp. 273-282.

[5] T. Germa, F. Lerasle, N. Ouadah, and V. Cadenat, "Vision and RFID data fusion for tracking people in crowds by a mobile robot," Computer Vision Image Understanding, vol. 114, no. 6, pp. 641-651, June 2010.

[6] K. Rohweder, P. Vorst, and A. Zell, "Improved mapping of RFID tags by fusion with spatial structure," in Proc. of the 4th European Conf. on Mobile Robots (ECMR 2009), Mlini/Dubrovnik, Croatia, September 2009, pp. 247-252.

[7] T. Deyle, H. Nguyen, M. Reynolds, and C. Kemp, "Rf vision: RFID receive signal strength indicator (RSSI) images for sensor fusion and mobile manipulation," in Proc. of the 2009 IEEE/RSJ Int. Conf. on Intelligent Robots and Systems (IROS 2009), St. Louis, MO, October 11-15 2009, pp. 5553-5560.

[8] J. S. Choi, H. Lee, D. Engels, and R. Elmasri, "Passive UHF RFIDbased localization using detection of tag interference on smart shelf," Systems, Man, and Cybernetics, Part C: Applications and Reviews, IEEE Transactions on, vol. 42, no. 2, pp. 268-275, March 2012.

[9] P. Vorst, A. Koch, and A. Zell, "Efficient self-adjusting, similaritybased location fingerprinting with passive UHF RFID," in Proc. of the 2011 IEEE Int. Conf. on RFID-Technology and Applications (RFID-TA 2011), Spain, September 2011, pp. 160-167.

[10] L. Ni, Y. Liu, Y. C. Lau, and A. Patil, "LANDMARC: indoor location sensing using active rfid," in Proc. of the 2003 IEEE Int. Conf. on Pervasive Computing and Communications (PerCom 2003), USA, March 2003, pp. 407-415.

[11] D. Fox, "Adapting the sample size in particle filters through KLDsampling," International Journal of Robotics Research, vol. 22, no. 12, pp. 985-1004, 2003.

[12] N. L. Johnson, S. Kotz, and N. Balakrishnan, Continuous Univariate Distributions. John Wiley \& Sons, vol. 1.

[13] W. Koch, "On exploiting negative sensor evidence for target tracking and sensor data fusion," Information Fusion, vol. 8, no. 1, pp. 28-39, 2007.

[14] J. Hoffmann, M. Spranger, D. Ghring, and M. Jngel, "Making use of what you dont see: Negative information in markov localization," in Proc. of the 2005 IEEE/RSJ Int. Conf. of Intelligent Robots and Systems (IROS 2005), August 2005, pp. 2947-2952.

[15] S. Thrun, D. Fox, W. Burgard, and F. Dellaert, "Robust monte carlo localization for mobile robots," Artificial Intelligence, vol. 128, no. 1-2, pp. 99-141, 2000. 